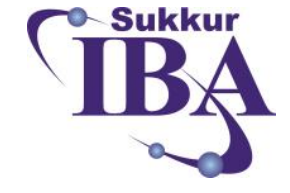

Vol. 1, No. 1 | Jan - June 2017

\title{
Unsteady Incompressible Stokes Flow through Porous Pipe of Uniform Circular Cross Section with Periodic Suction and Injection
}

\author{
Kaleemullah Bhatti \\ Department of Mathematics and Social Science, Sukkur IBA Sukkur, Pakistan \\ kaleem.msmts@iba-suk.edu.pk \\ Abdul Majeed Siddiqui \\ Department of Mathematics, Pennsylvania State University, York Campus \\ York, PA 17403, U.S.A. \\ ams5@psu.edu \\ Zarqa Bano \\ Department of Mathematics and Social Science, Sukkur IBA Sukkur, Pakistan \\ zarqa.bano@iba-suk.edu.pk
}

\begin{abstract}
This work is concerned with the flow of a Newtonian fluid through a pipe of constant circular cross section which is uniformly porous. The governing unsteady equations are solved analytically after converting them to a third order ordinary differential equation using similarity transformation method. Expressions for axial and radial velocity components and pressure distribution are obtained. The characteristics of complex axial velocity and complex radial velocity for different values of parameters are analysed. The effects of small suction and small injection are delineated through graphs. Results reveal that suction or injection has significant influence on the flow.
\end{abstract}

Keywords: Unsteady Stokes Flow, Porous pipe, circular cross section, Periodic Suction and Injection, No Slip.

\section{Introduction}

Stokes flows or creeping flows are gaining importance because of their wide range of applications. Such type of flows occurs in lubrication process, in swimming of microorganism or sperm, in the flow of lava, in paints and viscous polymers etc. There are many real world flow situations that correspond to the laminar flow through a channel with porous walls. One of the applications is in the modeling of process such as transpiration cooling, in which walls of the channel are protected from the heat by injection of cooler fluids; another application is to model the fluid flow occurring during separation of isotopes of uranium-235 and uranium-238 by gaseous diffusion in order to produce fuel for nuclear reactors. Another situation in which flow through porous channels occur is in the model to control the boundary layer flow of air on the wings of aircrafts by injection or suction of fluids into or out of the wings. Some of such situations require one or both the walls of the channel to be porous. 
Berman [1] (1953) was the first mathematician who encountered the problem of laminar flow through a porous channel of rectangular cross section. There was uniform injection through the lower plate and uniform suction through the upper plate. Narasimhan [6] (1961) extended the problem studied by Berman while considering flow of a nonNewtonian fluid through a uniform circular pipe. Khaled [5] (2004) studied the effect of slip conditions on Stokes and Couette flows due to oscillating wall. Ganesh [2] (2007) studied unsteady Stokes flow through a channel consisting of parallel porous plates when there is periodic suction through the lower plate and periodic injection through the upper plate. Zaman [4] (2013) considered the magneto-hydrodynamic fluid flow between parallel porous plates with uniform suction and injection. After that Kirubhashankar [3] (2014) studied the problem of unsteady magneto-hydrodynamic fluid flow through parallel porous plates when one plate was moving with constant velocity.

In this paper the flow of a Newtonian fluid through a uniformly porous pipe of constant circular cross is considered. The effects of small suction and small injection are delineated through graphs. Results reveal that suction or injection has significant influence on the flow.

\section{Problem Formulation}

We consider a circular pipe of uniform cross section and of infinite length and a Newtonian fluid passes through the pipe. The following flow assumptions are considered:

- Flow is axisymmetric with negligible body forces.

- Flow is unsteady.

- Stokes flow is presumed and therefore convective forces may be neglected in governing equations due to very small Reynolds Number.

- The walls of the cylindrical pipe are uniformly porous.
- The suction or injection occurs periodically through walls with velocity $v_{0} e^{i w t}$.

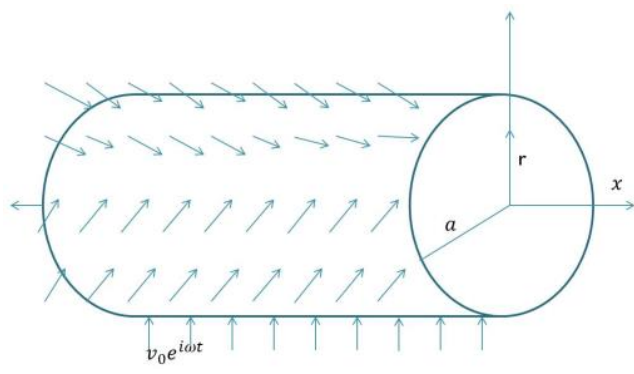

Fig. 1: Uniformly Porous Pipe with Circular Cross Section

We use cylindrical polar coordinates $(r, \theta, x)$ with $x$-axis as axial axis of pipe and $r$ as radial distance from the axis. Due to axial symmetry $\theta$ coordinate disappears throughout. Defining a dimensionless parameter $=\frac{r}{a},(0 \leq \xi \leq 1)$, where $a$ is radius of the pipe and we are going to introduce this parameter in flow equations and boundary conditions as follows:

$x$-Component:

$\frac{\partial u}{\partial t}=-\frac{1}{\rho} \frac{\partial p}{\partial x}+v\left(\frac{\partial^{2} u}{\partial x^{2}}+\frac{1}{a^{2}} \frac{\partial^{2} u}{\partial \xi^{2}}+\frac{1}{a^{2} \xi} \frac{\partial u}{\partial \xi}\right)$,

$r$-Component:

$\frac{\partial v}{\partial t}=-\frac{1}{a \rho} \frac{\partial p}{\partial \xi}+v\left(\frac{\partial^{2} v}{\partial x^{2}}+\frac{1}{a^{2}} \frac{\partial}{\partial \xi}\left(\frac{1}{\xi} \frac{\partial}{\partial \xi}(\xi v)\right)\right)$,

Continuity Equation:

$\frac{\partial}{\partial x}(\xi u)+\frac{1}{a} \frac{\partial}{\partial \xi}(\xi v)=0$,

where $u$ and $v$ are the velocity components in $x$ and $r$ direction in flow field at any 
time t. The boundary conditions of the flow at any instant $t$ are:

$$
\begin{aligned}
& u(x, 1)=0, \\
& \left(\frac{\partial u}{\partial \xi}\right)_{\xi=0}=0, \\
& v(x, 0)=0, \\
& v(x, 1)=v_{0} e^{i w t} .
\end{aligned}
$$

We chose velocity vector $\mathbf{U}$ and pressure $\mathrm{P}$ in the form:

$$
\begin{aligned}
& \overrightarrow{\mathbf{U}}=\{u(x, \xi) \hat{i}+v(x, \xi) \hat{j}\} e^{i \omega t}, \\
& P=p(x, \xi) e^{i \omega t}
\end{aligned}
$$

Using equation (8) and equation (9) the governing equations (1) to (3) reduce to:

$x$-Component:

$i w u=-\frac{1}{\rho} \frac{\partial p}{\partial x}$

$+v\left(\frac{\partial^{2} u}{\partial x^{2}}+\frac{1}{a^{2}} \frac{\partial^{2} u}{\partial \xi^{2}}+\frac{1}{a^{2} \xi} \frac{\partial u}{\partial \xi}\right)$,

$r$-Component:

$$
\begin{aligned}
& i w v=-\frac{1}{a \rho} \frac{\partial p}{\partial \xi} \\
& +v\left(\frac{\partial^{2} v}{\partial x^{2}}+\frac{1}{a^{2}} \frac{\partial}{\partial \xi}\left(\frac{1}{\xi} \frac{\partial}{\partial \xi}(\xi v)\right)\right),
\end{aligned}
$$

Continuity Equation:

$$
\frac{\partial}{\partial x}(\xi u)+\frac{1}{a} \frac{\partial}{\partial \xi}(\xi v)=0
$$

The boundary conditions reduce to:

$u(x, 1)=0$, $\left(\frac{\partial u}{\partial \xi}\right)_{\xi=0}=0$

$$
v(x, 0)=0,
$$

$v(x, 1)=v_{0}$.

\section{Solution to the problem}

Introducing the stream function $\psi$ as follows, so that the continuity equation (12) is identically satisfied:

$$
\begin{aligned}
& u(x, \xi)=\frac{1}{a^{2} \xi} \frac{\partial \psi}{\partial \xi}, \\
& v(x, \xi)=-\frac{1}{a \xi} \frac{\partial \psi}{\partial x} .
\end{aligned}
$$

Following the assumption of Berman [1] and Narasimhan [6], writing the stream function $\psi$ as:

$\psi(x, \xi)=g(x) h(\xi)$,

so that the velocities (17) and (18) are given as:

$$
\begin{aligned}
& u(x, \xi)=\frac{1}{a^{2} \xi} g(x) h^{\prime}(\xi), \\
& v(x, \xi)=-\frac{1}{a \xi} g^{\prime}(x) h(\xi) .
\end{aligned}
$$

The boundary conditions together with the knowledge of inlet conditions to the pipe give rise to an expression for $g(x)$.

$$
g(x)=\frac{1}{h(1)}\left(\frac{a^{2} u_{0}}{2}-a v_{0} x\right),
$$

Where $u_{0}$ is the average axial velocity of fluid at the entrance of pipe and is given as follows:

$u_{0}=2 \int_{0}^{1} \xi u(0, \xi) d \xi$.

Assuming that: 
K. Bhatti et al. Unsteady Incompressible Stokes Flow through Porous Pipe of Uniform Circular Cross Section with

$\phi(\xi)=\frac{h^{\prime}(\xi)}{\xi h(1)}$,

$\Phi(\xi)=\frac{h(\xi)}{\xi h(1)}=\frac{1}{\xi} \int_{0}^{\xi} t \phi(t) d t$

Using equations (24) and (25) we get the velocity components as follows:

$u(x, \xi)=\left(\frac{u_{0}}{2}-\frac{x v_{0}}{a}\right) \phi(\xi)$,

and

$v(x, \xi)=v_{0} \Phi(\xi)$,

and the stream function reduces to:

$\psi(x, \xi)=\left(\frac{a^{2} u_{0}}{2}-a v_{0} x\right) \xi \Phi(\xi)$

In above equations the function $\phi(\xi)$ is still to be determined. It is also worth to mention that radial velocity becomes function of $\xi$ only. Now using equations (26) and (27) in equations of motion (10) and (11) gives:

$$
\begin{aligned}
& -\frac{1}{\rho} \frac{\partial p}{\partial x}=\left(\frac{u_{0}}{2}-\frac{x v_{0}}{a}\right) \\
& \left(i w \phi(\xi)-\frac{v}{a^{2}}\left(\phi^{\prime \prime}(\xi)+\frac{\phi^{\prime}(\xi)}{\xi}\right)\right)
\end{aligned}
$$

and

$$
\begin{aligned}
& -\frac{1}{a \rho} \frac{\partial p}{\partial \xi}=i w v_{0} \Phi(\xi) \\
& -\frac{v v_{0}}{a^{2}}\left(\Phi^{\prime \prime}(\xi)+\frac{\Phi^{\prime}(\xi)}{\xi}-\frac{\Phi(\xi)}{\xi^{2}}\right) .(30)
\end{aligned}
$$

From onwards we would use the notations $\phi$ and $\Phi$ instead of $\phi(\xi)$ and $\Phi(\xi)$ in order to avoid repetition.
Differentiate equation (29) with respect to $\xi$ to get:

$$
\begin{aligned}
& -\frac{1}{\rho} \frac{\partial^{2} p}{\partial \xi \partial x}=\left(\frac{u_{0}}{2}-\frac{x}{a} v_{0}\right) \\
& \left(i w \phi^{\prime}-\frac{v}{a^{2}}\left(\phi^{\prime \prime \prime}+\frac{\phi^{\prime \prime}}{\xi}-\frac{\phi^{\prime}}{\xi^{2}}\right)\right),
\end{aligned}
$$

and differentiate equation (30) with respect to $x$ to get:

$$
-\frac{1}{\rho} \frac{\partial^{2} \rho}{\partial x \partial \xi}=0 .
$$

Equating the results of equation (31) equation (31) we get:

$\left(\frac{u_{0}}{2}-\frac{x}{a} v_{0}\right)\left(i w \phi^{\prime}-\frac{v}{a^{2}}\left(\phi^{\prime \prime \prime}+\frac{\phi^{\prime \prime}}{\xi}-\frac{\phi^{\prime}}{\xi^{2}}\right)\right)=0$

since this is to be satisfied for all $x$, therefore:

$i w \phi^{\prime}-\frac{v}{a^{2}}\left(\phi^{\prime \prime \prime}+\frac{\phi^{\prime \prime}}{\xi}-\frac{\phi^{\prime}}{\xi^{2}}\right)=0$.

Assuming $\quad \beta^{2}=\frac{\rho i w}{\mu} \quad$ equation becomes:

$\beta^{2} a^{2} \phi^{\prime}-\left(\phi^{\prime \prime \prime}+\frac{\phi^{\prime \prime}}{\xi}-\frac{\phi^{\prime}}{\xi^{2}}\right)=0$,

The boundary conditions on functions $\phi$ and $\Phi$ can be obtained using equations (26) and (27) and already prescribed boundary conditions (13) to (16) and are given below:

$\phi(1)=0, \phi^{\prime}(0)=0, \Phi(0)=0, \Phi(1)=1 .(36)$ 
The third order linear ODE (35) together with the associated boundary (36) constitute an exact solution to equations of motion and continuity as formulated. Equation (35) is well known Modified Bessel's equation with order " $a \beta$ ". The general solution of equation (35) is easily obtained in terms of Modified Bessel functions as below:

$\phi(\xi)=c_{1}+c_{2} I_{0}(a \beta \xi)+c_{3} K_{0}(a \beta \xi)$

where $I_{\alpha}(x)$ and $K_{\alpha}(x)$ are Modified Bessel functions of first and second kind respectively and are defined as follows:

$$
\begin{aligned}
& I_{\alpha}(x)=\sum_{m=0}^{\infty} \frac{1}{m ! \Gamma(m+\alpha+1)}\left(\frac{x}{2}\right)^{2 m+\alpha}, \\
& K_{\alpha}(x)=\frac{\pi}{2} \frac{I_{-\alpha}(x)-I_{\alpha}(x)}{\sin (\pi \alpha)} .
\end{aligned}
$$

Since $1, I_{0}(a \beta \xi)$ and $K_{0}(a \beta \xi)$ are three linearly independent solutions, therefore we can drop $K_{0}(a \beta \xi)$, having less contribution in solution. Also $K_{0}(a \beta \xi)$ diverges at $\xi=0 \quad$ with singularity of logarithmic type. With this assumption equation (37) reduces to:

$$
\phi(\xi)=c_{1}+c_{2} I_{0}(a \beta \xi) .
$$

Using substitution (25) and boundary conditions (36) we can easily find the values of arbitrary constants as given below:

$$
\begin{aligned}
& c_{1}=\frac{2 a \beta I_{0}(a \beta)}{a \beta I_{0}(a \beta)-2 I_{1}(a \beta)}, \\
& c_{2}=\frac{-2 a \beta}{a \beta I_{0}(a \beta)-2 I_{1}(a \beta)} .
\end{aligned}
$$

Substituting these constants in general solution (40) we get:

$\phi(\xi)=\frac{2 a \beta\left(I_{0}(a \beta)-I_{0}(a \beta \xi)\right)}{a \beta I_{0}(a \beta)-2 I_{1}(a \beta)}$,

and using equation (25)

$$
\Phi(\xi)=\frac{a \beta \xi I_{0}(a \beta)-2 I_{1}(a \beta \xi)}{a \beta I_{0}(a \beta)-2 I_{1}(a \beta)} .
$$

Substitute $\phi(\xi)$ and $\Phi(\xi)$ in the components of velocity defined in equations (26) and (27) to get:

$u(x, \xi)=\left(\frac{u_{0}}{2}-\frac{x v_{0}}{a}\right) \phi(\xi)$

$u(x, \xi)=\left(\frac{u_{0}}{2}-\frac{x v_{0}}{a}\right) \frac{2 a \beta\left(I_{0}(a \beta)-I_{0}(a \beta \xi)\right)}{a \beta I_{0}(a \beta)-2 I_{1}(a \beta)}$,

and

$v(x, \xi)=v_{0} \Phi(\xi)$

$v(x, \xi)=v_{0} \frac{a \beta \xi I_{0}(a \beta)-2 I_{1}(a \beta \xi)}{a \beta I_{0}(a \beta)-2 I_{1}(a \beta)}$.

Using equation (46) in equation (8), unsteady components of velocity are: 


$$
\begin{aligned}
& u(x, \xi, t)=\left(\frac{u_{0}}{2}-\frac{x v_{0}}{a}\right) \\
& \frac{2 a \beta\left(I_{0}(a \beta)-I_{0}(a \beta \xi)\right)}{a \beta I_{0}(a \beta)-2 I_{1}(a \beta)} e^{i w t},
\end{aligned}
$$

and

$$
v(x, \xi, t)=v_{0} \frac{a \beta \xi I_{0}(a \beta)-2 I_{1}(a \beta \xi)}{a \beta I_{0}(a \beta)-2 I_{1}(a \beta)} e^{i w t} .
$$

Equations (47) and (48) now fully define the axial and radial velocity components respectively.Further introducing the following non-dimensional parameters:

$$
N_{R E}=\frac{a u_{0}}{v}
$$

$R=\frac{a v_{0}}{v}$

equations (47) and (48) can be written in non-dimensional form as follows:

$$
\begin{aligned}
& \frac{u(x, \xi, t)}{u_{0}}=\left(\frac{1}{2}-\frac{x}{a} \frac{R}{N_{R E}}\right) \\
& \frac{2 a \beta\left(I_{0}(a \beta)-I_{0}(a \beta \xi)\right)}{a \beta I_{0}(a \beta)-2 I_{1}(a \beta)} e^{i w t},
\end{aligned}
$$

and

$$
\frac{v(x, \xi, t)}{v_{0}}=\frac{a \beta \xi I_{0}(a \beta)-2 I_{1}(a \beta \xi)}{a \beta I_{0}(a \beta)-2 I_{1}(a \beta)} e^{i w t}
$$

The velocity field is now fully defined by equation (51) for axial component and by equation (52) for radial component.

\section{Pressure Distribution}

The pressure distribution can be obtained by extracting the pressure gradients from (29) and (30) and by integrating with respect to $x$ and $\xi$ respectively. Thus:

$\int_{0}^{x} \frac{\partial p}{\partial x} d x=p(x, \xi)-p(0, \xi)$,

$\int_{0}^{\xi} \frac{\partial p}{\partial \xi} d \xi=p(x, \xi)-p(x, 0)$.

It follows from (53) and (54) that:

$\int_{0}^{x} \frac{\partial p}{\partial x} d x+\left(\int_{0}^{\xi} \frac{\partial p}{\partial \xi} d \xi\right)_{x=0}=p(x, \xi)-p(0,0)$

Using (29) and (30) we get:

$p(x, \xi)=p(0,0)+$

$\int_{0}^{\xi} \mu\left(-a \beta^{2} v_{0} \Phi(\xi)+\frac{v_{0}}{a}\left(\frac{\mathrm{d}^{2}}{\mathrm{~d} \xi^{2}} \Phi(\xi)+\frac{\frac{\mathrm{d}}{\mathrm{d} \xi} \Phi(\xi)}{\xi}-\frac{\Phi(\xi)}{\xi^{2}}\right) \mathrm{d} \xi+\right.$

$\frac{1}{2} \frac{\mu v_{0} x^{2}}{a}\left(\beta^{2} \phi(\xi)-\frac{1}{a^{2}}\left(\frac{\mathrm{d}^{2}}{\mathrm{~d} \xi^{2}} \phi(\xi)+\frac{\frac{\mathrm{d}}{\mathrm{d} \xi} \phi(\xi)}{\xi}\right)\right)-$

$\frac{1}{2} \mu u_{0}\left(\beta^{2} \phi(\xi)-\frac{1}{a^{2}}\left(\frac{\mathrm{d}^{2}}{\mathrm{~d} \xi^{2}} \phi(\xi)+\frac{\frac{\mathrm{d}}{\mathrm{d} \xi} \phi(\xi)}{\xi}\right)\right) x$,

and hence from equation (9)

$P(x, \xi, t)=p(x, \xi) e^{i \omega t}$

where $p(0,0)$ is pressure at the entrance of the channel. One can easily complete the calculations for pressure distribution by making the substitutions for $\phi(\xi)$ and 
K. Bhatti et al. Unsteady Incompressible Stokes Flow through Porous Pipe of Uniform Circular Cross Section with

$\Phi(\xi)$ from equations (43) and (44) in the equation (56) and (57).

\section{Results and Discussion}

In this chapter the expressions for axial and radial velocity components for the two dimensional unsteady stokes flow through a pipe of uniform cross section and radius are obtained in equations (51) and (52). The case when $0<\mathrm{R} \leq 1$, where denotes wall Reynolds number, corresponds to small suction because wall velocity is taken positive in positive direction i.e., for suction. Hence if there is small suction through the porous pipe it can be clearly seen that the axial velocity decreases as fluid moves forward in the pipe in positive direction, see figures 2, 3, 4 and 8. The results may be justified with the argument that due to small suction internal pressure has been decreased.

The case when $-1 \leq \mathrm{R}<0$ corresponds to small injection. and both are negative in the negative direction. Hence if the axial velocity increases as fluid moves forward in the pipe in positive direction, see figures 5, 6, 7 and 9. From figure $2-7$ one can observe that the axial velocity completely vanishes when and behaves like a constant near the axis of the pipe i.e, from to and almost vanishes near the wall i.e, when $\xi=1$.

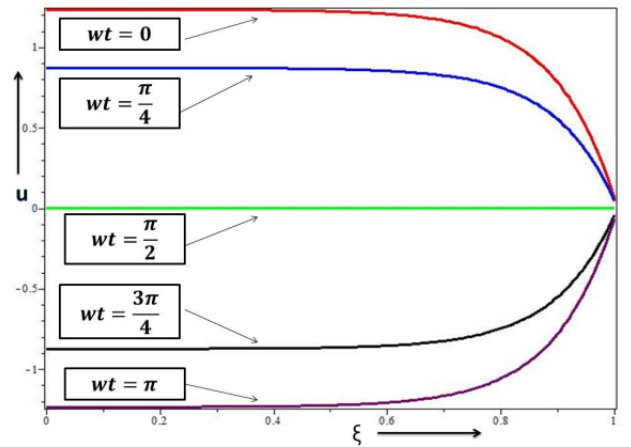

Figure 2: Axial velocity profile for small suction $R=1 x=0, a=10, \beta=1 N_{R E}=10$, for few distinct values of $w t$

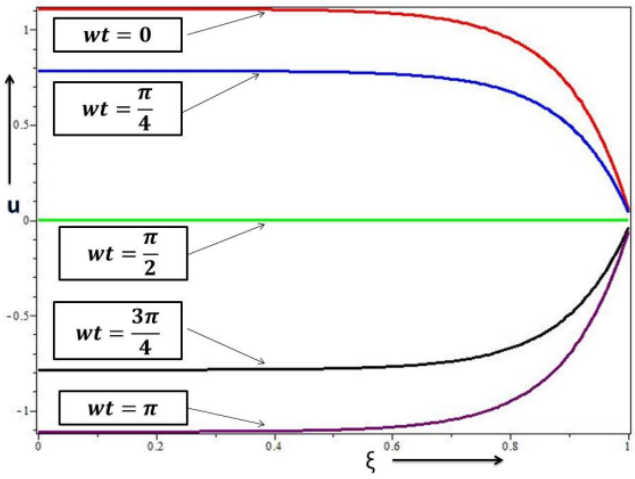

Figure 3: Axial velocity profile for small suction $R=1 x=5, a=10, \beta=1 N_{R E}=10$, for few distinct values of $w t$

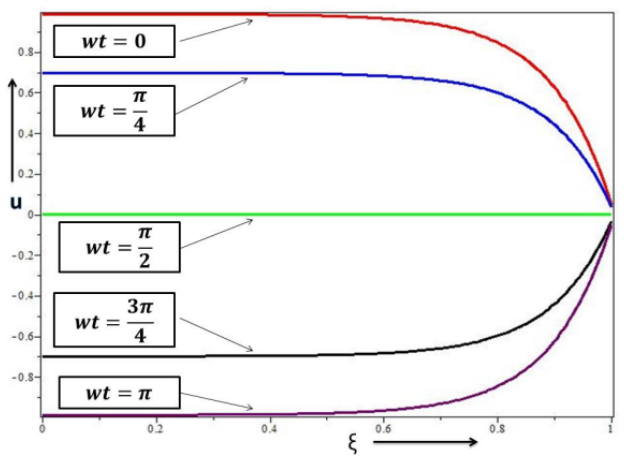

Figure 4: Axial velocity profile for small suction $R=1 x=10, a=10, \beta=1 N_{R E}=10$, for few distinct values of $w t$

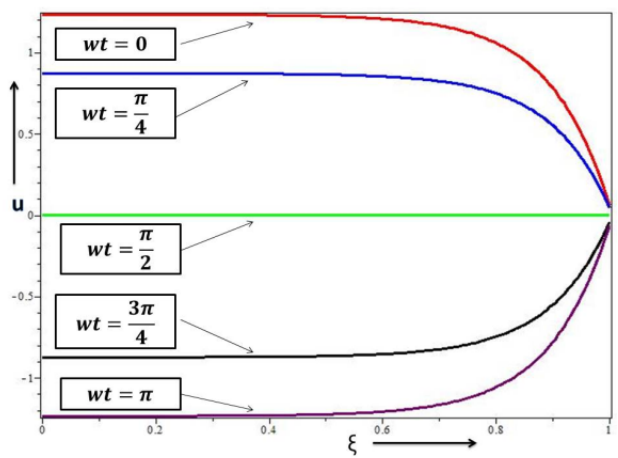

Figure 5: Axial velocity profile for small injection 
K. Bhatti et al. Unsteady Incompressible Stokes Flow through Porous Pipe of Uniform Circular Cross Section with

$R=-1 \quad x=0, a=10, \quad \beta=1 \quad N_{R E}=10 \quad R=1, \quad a=10, \quad \beta=1 \quad N_{R E}=10$,

, for few distinct values of $w t$

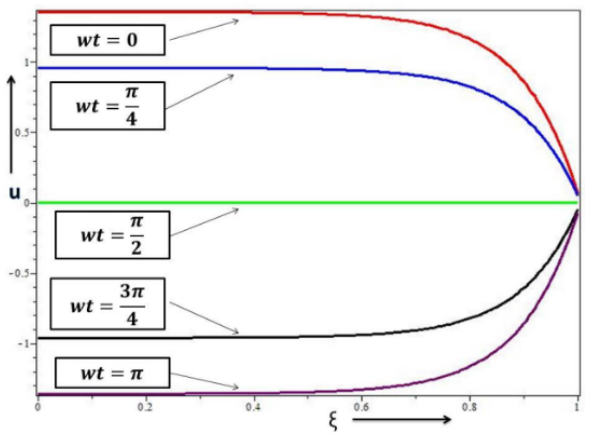

Figure 6: Axial velocity profile for small injection

$R=-1 \quad x=5, a=10, \beta=1 N_{R E}=10$

, for few distinct values of wt

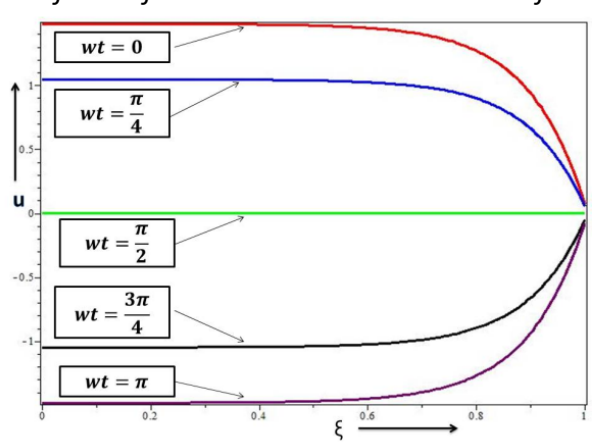

Figure 7: Axial velocity profile for small injection

$R=-1 \quad x=10, \quad a=10, \quad \beta=1$

$N_{R E}=10$, for few distinct values of $w t$

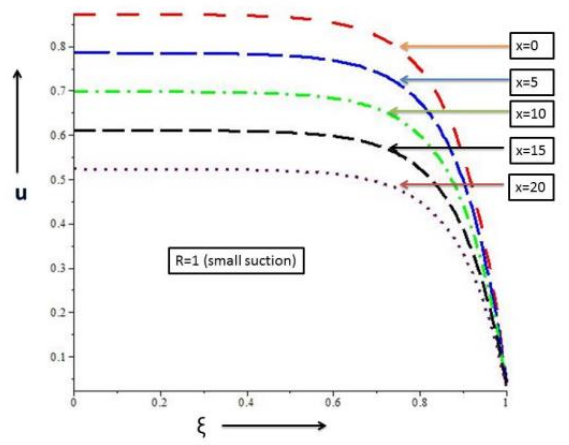

Figure 8: Axial velocity profile for small suction $w t=\frac{\pi}{4}$ at few distinct cross sections of the

pipe

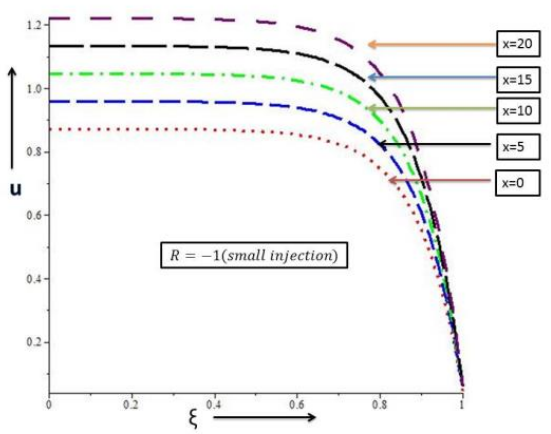

Figure 9: Axial velocity profile for small injection $R=-1, \quad a=10, \quad \beta=1 \quad N_{R E}=10$, $w t=\frac{\pi}{4}$ at few distinct cross sections of the pipe

\section{Conclusion}

Unsteady stokes flow of a Newtonian fluid past a pipe of uniform cross section have been discussed analytically, when there is periodic suction or periodic injection through its porous surface. The expressions for axial and radial velocity components and pressure distribution have been derived and analysed graphically for two cases i.e, for small suction and for small injection, depending on values of wall Reynolds number. It has been found that in case if there is small suction, the magnitude of axial velocity decreases and it increases if there is small injection.

\section{References}

[1]. S. Berman, "Laminar Flow in Channels with Porous Walls" in J. of Appl. Physics vol.24, pp. 1232, 1953.

[2]. S. Ganesh, "Unsteady Stokes Flow of Viscous Fluid Between Two Parallel Porous Plates" in J. of Inf. Sci. Comp. vol.1 no.1, pp63, 2007. 
K. Bhatti et al. Unsteady Incompressible Stokes Flow through Porous Pipe of Uniform Circular Cross Section with

[3]. K. Kirubhashankar, "An Exact

Solution of the Problem of Unsteady MHD

Flow through Parralel Porous Plates" in Int. J. of Adv. in Mech. Automobile Engg, vol.1, issue.1, pp39, 2014.

[4]. H. Zaman, "Hall Effects on the Unsteady Incompressible MHD Fluid Flow with Slip Conditions and Porous Walls" in Applied Math. and Phy. vol.1, no.2, pp.31, 2013.

[5]. A. R. A. Khaled, "The Effect of Slip Condition on Stokes and Coutte Flows due to an Oscillating Wall: Exact Solutions" in Int. J. Non-Linear Mech., vol.39, pp795, 2004.

[6]. M. N. L. Narasimhan, "Laminar Non-Newtonian Flow in a Porous Pipe" in Appl. Sci. Res. vol.10, Sec A, 1961.

[7]. N. M. Bujurke, "Laminar Flow In A Uniformly Porous Pipe"in Indian J. Pure Appl. Math. vol.31 no.3, pp341, 2000. 